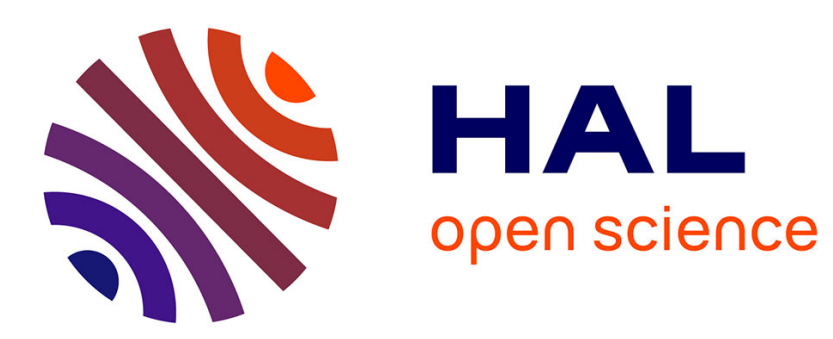

\title{
Realization of Harmonic Oscillator Arrays with Graded Semiconductor Quantum Wells
}

C. Deimert, P. Goulain, J.-M. Manceau, W. Pasek, T. Yoon, A. Bousseksou, N. Y. Kim, R. Colombelli, Z. R. Wasilewski

\section{- To cite this version:}

C. Deimert, P. Goulain, J.-M. Manceau, W. Pasek, T. Yoon, et al.. Realization of Harmonic Oscillator Arrays with Graded Semiconductor Quantum Wells. Physical Review Letters, 2020, 125 (9), pp.097403. 10.1103/PhysRevLett.125.097403 . hal-03011984

\section{HAL Id: hal-03011984 \\ https://hal.science/hal-03011984}

Submitted on 23 Nov 2020

HAL is a multi-disciplinary open access archive for the deposit and dissemination of scientific research documents, whether they are published or not. The documents may come from teaching and research institutions in France or abroad, or from public or private research centers.
L'archive ouverte pluridisciplinaire HAL, est destinée au dépôt et à la diffusion de documents scientifiques de niveau recherche, publiés ou non, émanant des établissements d'enseignement et de recherche français ou étrangers, des laboratoires publics ou privés. 


\title{
Realization of Harmonic Oscillator Arrays with Graded Semiconductor Quantum Wells
}

\author{
C. Deimert $\odot,{ }^{1}$ P. Goulain $\odot,{ }^{2}$ J.-M. Manceau, ${ }^{2}$ W. Pasek $\odot,{ }^{1,3}$ T. Yoon $\odot,{ }^{1,3}$ A. Bousseksou, ${ }^{2}$ N. Y. Kim $\odot,{ }^{1,3,4,5,6}$ \\ R. Colombelli®, ${ }^{2, *}$ and Z. R. Wasilewski® ${ }^{1,3,4,5, \dagger}$ \\ ${ }^{1}$ Department of Electrical and Computer Engineering, University of Waterloo, \\ 200 University Avenue West, Waterloo, Ontario N2L 3G1, Canada \\ ${ }^{2}$ Centre de Nanosciences et de Nanotechnologies (C2N), CNRS UMR 9001, Université Paris-Saclay, 91120 Palaiseau, France \\ ${ }^{3}$ Institute for Quantum Computing, University of Waterloo, 200 University Avenue West, Waterloo, Ontario N2L 3G1, Canada \\ ${ }^{4}$ Waterloo Institute for Nanotechnology, University of Waterloo, 200 University Avenue West, Waterloo, Ontario N2L 3G1, Canada \\ ${ }^{5}$ Department of Physics and Astronomy, University of Waterloo, 200 University Avenue West, Waterloo, Ontario N2L 3G1, Canada \\ ${ }^{6}$ Department of Chemistry, University of Waterloo, 200 University Avenue West, Waterloo, Ontario N2L 3G1, Canada
}

(Received 3 January 2020; accepted 28 July 2020; published 28 August 2020)

\begin{abstract}
The harmonic oscillator is a foundational concept in both theoretical and experimental quantum mechanics. Here, we demonstrate harmonic oscillators in a semiconductor platform by faithfully implementing continuously graded alloy semiconductor quantum wells. Unlike current technology, this technique avoids interfaces that can hamper the system and allows for the production of multiwell stacks several micrometers thick. The experimentally measured system oscillations are at $3 \mathrm{THz}$ for two structures containing 18 and 54 parabolic quantum wells. Absorption at room temperature is achieved: this is as expected from a parabolic potential and is unlike square quantum wells that require cryogenic operation. Linewidths below $11 \%$ of the central frequency are obtained up to $150 \mathrm{~K}$, with a $5.6 \%$ linewidth obtained at $10 \mathrm{~K}$. Furthermore, we show that the system correctly displays an absence of nonlinearity despite electronelectron interactions - analogous to the Kohn theorem. These high-quality structures already open up several new experimental vistas.
\end{abstract}

DOI: 10.1103/PhysRevLett.125.097403

Harmonic oscillators are ubiquitous in physics. Pendulums, vibrational modes in molecules, resonant electrical circuits, and many other systems can be modeled as harmonic oscillators. Even tools like Fourier transforms and second quantization have their roots in the mathematics of harmonic oscillation. With such wide-ranging usage, implementing harmonic oscillators in the real world-as faithfully as possible-remains a compelling challenge.

For quantum harmonic oscillators, some prominent techniques exist, including parabolic optical traps and Landau levels in cyclotron systems. However, semiconductor heterostructures are an attractive platform because band gap engineering allows for near-direct control over the shape of the nanometer-scale parabolic potential. Such systems are interesting from both a theoretical and practical standpoint. One striking feature is that their oscillation frequency is almost completely governed by the bare parabolic potential. Electron-electron interactions cancel out-similar to the Kohn theorem in cyclotron systems [1] - leaving the resonance frequency almost completely independent of the charge distribution [2]. Besides its elegance, this result means that semiconductor parabolic quantum wells are remarkably robust to thermal charge fluctuations.

This feat is particularly relevant in terahertz optoelectronics, which is the subject of intense research in view of applications in communications, medicine, security, and fundamental science [3]. Thermal charge fluctuations play a key role in these devices - usually a detrimental one. One of the most promising emitters, the terahertz quantum cascade laser, has long struggled to reach operating temperatures above $200 \mathrm{~K}[4,5]$. The situation for detectors is even worse, with terahertz quantum well infrared photodetectors failing above $25 \mathrm{~K}$ [6].

Semiconductor parabolic quantum wells (PQWs) have already shown early promise in the terahertz range. Recently, there has been growing interest to push terahertz devices into the strong-coupling regime [7], where one can envision new sources of coherent [8] or nonclassical [9] light. Square quantum wells are inadequate here, because they exhibit a serious degradation of the absorption resonance above cryogenic temperatures [10]. Although the thermal degradation can be mitigated through the use of collective modes [11], this requires heavy doping levels, which lead to impurity scattering and strong plasma blueshifts that are especially troublesome in the terahertz regime. So, the fact that terahertz parabolic potentials have a transition independent on charge distribution is a key advantage here, and there is already preliminary evidence of room temperature strong coupling in semiconductor systems [12].

Growing high-quality PQWs is challenging, however, and this has limited their application. Most studies of 

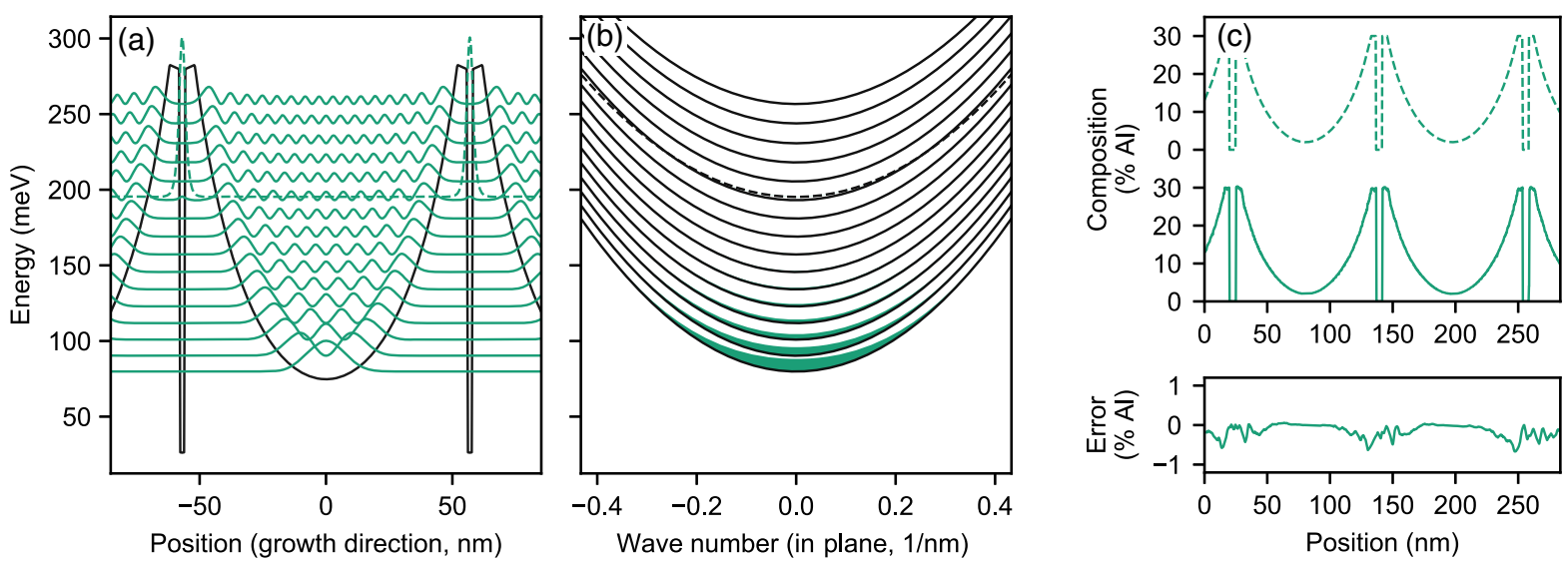

FIG. 1. (a) Schrodinger-Poisson simulation of a periodic stack of PQWs at $300 \mathrm{~K}$, performed with nextnano. The bare (undoped) PQW intersubband transition energy is $\sim 3 \mathrm{THz}$. Modulation doping of $1 \times 10^{11} \mathrm{~cm}^{-2}$ per well is placed in 2 nm square wells in between the PQWs. The black curve shows the conduction band edge, while the green curves show the squared envelope wave functions, shifted according to their subband energy. (b) In-plane subband dispersions of the same simulation. Shaded regions represent the relative electron occupation. In both (a) and (b), the dashed lines correspond to the bound state of the square doping well. (c) Top: target composition (dashed) and measured composition (solid), based on direct flux measurements from the Al cell, using an ion gauge.

(c) Bottom: shows the difference between the two curves.

PQWs took place at the end of the 1980s, focusing on their structural analysis [13] or on 2D electronic gases for transport applications [14-17]. More recently, the interband optical properties have been studied in undoped structures [18]. However, the majority of these works only approximated the parabolic potentials via digital alloys. While some studies used smoothly graded wells, the techniques were difficult to scale up accurately for the large PQW arrays needed for terahertz devices. To date, the terahertz optical response of PQWs has only been studied in digital alloys [12,19], with intersubband (ISB) linewidths limited to $\geq 10 \%$, even at $4 \mathrm{~K}$.

In this Letter, we demonstrate arrays of harmonic oscillators in a semiconductor platform by faithfully implementing true parabolic potentials with continuously graded $\mathrm{Al}_{x} \mathrm{Ga}_{1-x}$ As alloy semiconductor quantum wells (QWs). The system shows robust harmonic oscillator behavior at $3 \mathrm{THz}$ up to room temperature. Linewidths below $11 \%$ are obtained up to $150 \mathrm{~K}$ (with a $5.6 \%$ linewidth obtained at $10 \mathrm{~K}$ ), and the resonance frequency is stable with respect to both temperature and doping variations.

We grew two PQW array systems-one with a stack of 54 PQWs with $1 \times 10^{11} \mathrm{~cm}^{-2}$ electrons per well and the other with a stack of $18 \mathrm{PQWs}$ with $3 \times 10^{11} \mathrm{~cm}^{-2}$ electrons per well. The wells were realized in an $\mathrm{Al}_{x} \mathrm{Ga}_{1-x}$ As material system by varying the $\mathrm{Al}$ composition $x$ in the 2\%-30\% range along the growth direction [Figs. 1(a) and 1(c)]. The Al composition was controlled during growth by careful compensation of the Al cell's thermal dynamics [20] [Fig. 1(c)]. With this method, we can achieve precise control over the Al composition at standard growth rates $>0.15 \mathrm{~nm}$ and with substrate rotation to mitigate nonuniformity across the wafer. Unlike feedback-loop-based approaches (e.g., Ref. [21]), our method does not require real-time flux measurement during growth. The PQWs were each $103.8 \mathrm{~nm}$ wide, separated by $10 \mathrm{~nm}$ barriers comprising 4/2/4 $\mathrm{nm}$ of $\mathrm{Al}_{0.3} \mathrm{Ga}_{0.7} \mathrm{As} / \mathrm{GaAs} / \mathrm{Al}_{0.3} \mathrm{Ga}_{0.7} \mathrm{As}$. Si doping was placed in the GaAs region in the center of the barrier.

To analyze the quality of the PQW stacks, we measured the terahertz transmission with a Fourier spectrometer (details in the Supplemental Material [22]). The results are reported in Fig. 2. An absorption resonance is observed in both samples near $3 \mathrm{THz}$; it is already very clear at room temperature and narrows further as the samples are cooled. The absorption behavior is consistent with a strong, unified QW resonance and minimal inhomogeneous broadening. To extract more quantitative information, we fitted the data with an empirical model. We used a single-mode form of the dielectric tensor for the PQW stack, with the growthdirection $(z)$ component as [23]

$$
\frac{1}{\epsilon_{z z}(\omega)}=\frac{1}{\epsilon_{s}}\left(1+\frac{\omega_{P}^{2}}{\omega^{2}-\omega_{0}^{2}+i \gamma \omega}\right) .
$$

Here $\epsilon_{s}\left(\approx \epsilon_{x x}=\epsilon_{y y}\right)$ is the average static dielectric constant of the PQW stack. The parameters $\omega_{0}, \gamma$, and $\omega_{P}$ can be intuitively understood as the resonant frequency, homogeneous linewidth, and plasma frequency, respectively, but they are treated here as parameters to be fitted. This dielectric tensor is fed into a transfer matrix model to calculate the power transmission through the sample. In the limits of long wavelength (thin active region) and low absorption, this approach reduces to a simpler exponentially decaying power rule [24]. While the exponential decay model is commonly employed in multipass absorption, here the full transfer matrix approach is necessary to 

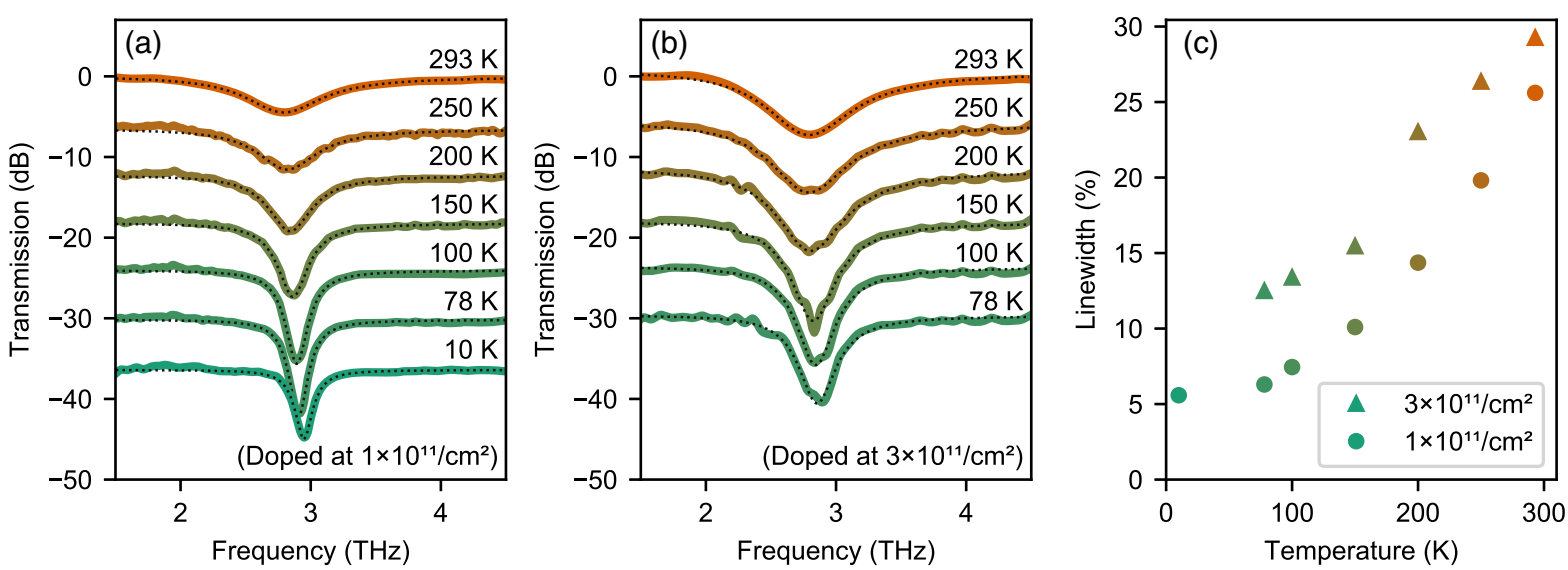

FIG. 2. Transmission measurements for the two PQW samples over a range of temperatures. (a) $54 \mathrm{PQWs}$ doped at $1 \times 10^{11} \mathrm{~cm}^{-2}$ per well. (b) $18 \mathrm{PQWs}$ doped at $3 \times 10^{11} \mathrm{~cm}^{-2}$ per well. In both (a) and (b), solid lines show the measured transmission spectra, and dotted lines show the empirical fit. Measurements at different temperatures are offset by $6 \mathrm{~dB}$ for clarity. (c) Linewidth $\gamma$ as a function of temperature. $\gamma$ was extracted from fits of the transmission data and it is expressed as a percentage of the fitted center frequency $\omega_{0}$.

explain the apparent asymmetry of the peaks, which arises due to variations in the electric field overlap with the PQW stack as a function of wavelength.

The experimentally extracted linewidths $\gamma$ are shown in Fig. 2(c) as a function of temperature. They are consistently smaller than $30 \%$ of the transition frequency, reaching just $5.6 \%$ for the lighter-doped, 54-well sample at $10 \mathrm{~K}$. Remarkably, for this sample, $\gamma$ remains below $11 \%$ right up to $150 \mathrm{~K}$, while the integrated absorption is nearly constant with temperature [Fig. 3(a)]. This is in contrast to terahertz transitions in square QWs, which experience both significant broadening and a sharp dropoff of the fundamental absorption peak with temperature. Of particular interest for experimental applications is the region $77-150 \mathrm{~K}$, wherein these PQWs exhibit linewidths better than $11 \%$, providing a high-quality terahertz resonance above liquid nitrogen temperatures.

Another notable feature of the absorption data is the stability of the resonant frequency $\omega_{0}$, which varies only from 2.8 to $3.0 \mathrm{THz}$ across all the temperatures and doping levels that we have studied. The stability of the peak position with doping, in particular, provides an elegant demonstration of the results of Ref. [2], similar to the Kohn theorem for cyclotron systems.

Understanding this and more fully modeling the absorption behavior of these PQWs is a subtle task. At the doping levels studied here, the Hartree potential leads to a significant redshift of the ISB transition energy, on the order of several meV. Furthermore, over most of the temperatures and doping levels studied, several subbands are occupied, which would seemingly preclude the use of a single-resonator model like Eq. (1).

To explain the simplicity and stability of our observed absorption peaks, we must go beyond the single-electron approximation and include interactions between the different ISB plasmon modes. In this case, a QW can still be modeled with an effective permittivity tensor, with a growth-direction component of the form

$$
\frac{1}{\epsilon_{z z}(\omega)}=\frac{1}{\epsilon_{s}}\left(1+\sum_{j} \frac{\Omega_{P, j}^{2}}{\omega^{2}-\Omega_{j}^{2}+i \Gamma_{j} \omega}\right),
$$

Here $\Omega_{P, j}, \Omega_{j}$, and $\Gamma_{j}$ are the weights, resonance frequencies, and homogeneous linewidths, respectively, of the absorption modes. However, these are now collective "multisubband" plasmon modes that arise from the interaction between different ISB transitions [11]. The weights and resonance frequencies can be calculated using either a quantum approach [27] or a semiclassical approach [28], which are equivalent in the long-wavelength limit.

In the case of a PQW, such modeling predicts that almost all of the absorption strength should be concentrated in a single mode $j$, whose frequency $\Omega_{j}$ is locked to the bare ISB transition of the undoped well-regardless of doping and temperature [11]. This notable behavior of harmonic systems is exactly what we see experimentally in Fig. 2.

While the overall behavior is as expected, a more precise comparison between model and theory illuminates lingering uncertainties. The composition profile of the grown structures was designed using next [29]—a Schrödinger-Poisson solver - to give a perfectly quadratic conduction band in the absence of doping. The full model (quantum or semiclassical) then predicts that this composition profile should give a stable absorption resonance at around $3.3 \mathrm{THz}$ - shown for the 54-well sample in the solid lines of Fig. 3(b).

This predicted absorption frequency is around $0.3 \mathrm{THz}$ higher than what is experimentally observed. One possible explanation for this discrepancy is the dependence of the $\mathrm{Al}_{x} \mathrm{Ga}_{1-x} \mathrm{As}$ band gap on composition. Nextnano uses, by default, the cubic dependence recommended by 

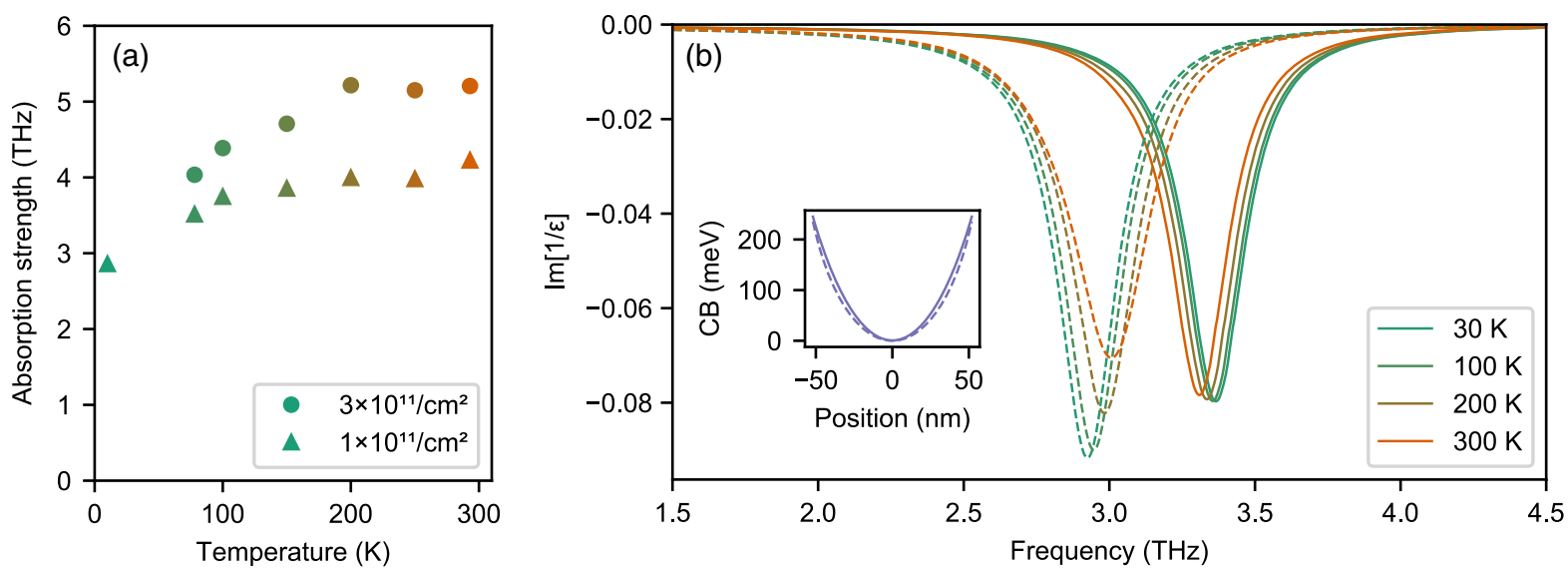

FIG. 3. (a) Measured absorption strength of the doped samples. Absorption strength here is defined as $\omega_{P} \sqrt{N_{\mathrm{QW}}}$, where $\omega_{P}$ is extracted by fitting the data with Eq. (1), and $N_{\mathrm{QW}}$ is the number of wells in the sample. (b) Calculated absorption spectra for the sample doped at $1 \times 10^{11} \mathrm{~cm}^{-2}$. Absorption is quantified by $\operatorname{Im}[1 / \varepsilon(\omega)]$, using Eq. (2). A constant, arbitrarily chosen $\Gamma_{j}=1 \mathrm{meV}$ was used to isolate the multisubband plasmon effects. The composition profile is assumed to follow that of the grown sample. However, two different relationships are used for the $\mathrm{Al}_{x} \mathrm{Ga}_{1-x} \mathrm{As}$ band gap vs composition. The solid curves correspond to the cubic dependence recommended by [25]. The dashed lines correspond to the linear dependence of [26]. In both cases, a 60:40 split was used between conduction and valence band offsets. The inset shows the two $\mathrm{CB}$ edge profiles vs position in the growth direction.

Ref. [25]. However, careful measurements of $\mathrm{Al}_{x} \mathrm{Ga}_{1-x} \mathrm{As}$ composition and band gap show that a linear dependence would be more appropriate for $x<0.45$ [26]. This distinction amounts to a minor correction for QWs with single-composition barriers, but could have a significant impact on smoothly graded PQWs, as clarified in Fig. 3(b). A relatively small deviation in the conduction band edge [Fig. 3(b), inset] widens the PQW and leads to a significant shift in the peak position. We see that the band gap dependence of Ref. [26] predicts a peak position close to what is experimentally measured.

This issue of the conduction band edge dependence on composition should appear whether doping is present or not. So, to further characterize the PQWs, we performed photoluminescence excitation (PLE) measurements on undoped samples at $\sim 10 \mathrm{~K}$. In this case, a stack of 10 undoped PQWs was grown using otherwise identical growth conditions as the doped samples. The $2 \mathrm{~nm}$ GaAs wells were skipped so that the barriers were $8 \mathrm{~nm}$ of $\mathrm{Al}_{0.30} \mathrm{Ga}_{0.70}$ As.

The PLE measurements provide a series of peaks corresponding to the $\mathrm{CB}_{n} \rightarrow \mathrm{HH}_{m}$ transitions ( $n$ and $m$ being subband indices). For PQWs like these, we expect to see an evenly spaced ladder of subbands in both the conduction (CB) and heavy hole (HH) bands. So, from the series of PLE peaks, we can extract the spacing between adjacent subbands. After accounting for exciton binding energies using a variational approach [30], we found subband spacings of $\Delta E_{\mathrm{HH}} / h=1.1$ and $\Delta E_{\mathrm{CB}} / h=3.0 \mathrm{THz}$. (Further details are provided in the Supplemental Material [22].)

These results are in good agreement with eight-band $\mathbf{k} \cdot \mathbf{p}$ modeling in nextnano, as long as the band gap dependence of Ref. [26] is used. Moreover, the CB subband spacing extracted from PLE is consistent with the absorption resonance of the $1 \times 10^{11} \mathrm{~cm}^{-2}$-doped sample at $10 \mathrm{~K}$ [Fig 2(a)]. This agreement between the absorption results and the bare ISB frequency calculated by PLE further supports the result of Ref. [2] that the PQW absorption frequency is independent of the number or distribution of electrons. It would, further, seem to suggest that the band gap dependence of Ref. [26] is closer to the correct one-however, other discrepancies still need to be addressed. For example, the absorption resonances based on Ref. [26] [Fig. 3(b), dashed lines] predict a blueshift with temperature, while Figs. 2(a) and 2(b) show a clear redshift.

One possible explanation for this is our use of the effective mass approximation in the calculation of Fig. 3. The approaches of Refs. [27,28] require a well-defined effective mass that is the same for each subband. This assumption cannot be made for PQWs in $\mathrm{Al}_{x} \mathrm{Ga}_{1-x}$ As: the effective mass for each subband is different, as the electrons in higher subbands see a larger aluminum composition on average. The full dispersion of each subband needs to be accounted for, and the transition currents should be defined in a manner that is compatible with the $\mathbf{k} \cdot \mathbf{p}$ method.

Initial approaches that have been employed to account for this [31] implicitly rely on the assumption that the overlap length of the ISB transition currents is constant. This is approximately true for square QWs, but it does not hold for other quantum well shapes [11]. For PQWs, where the wave function spread increases substantially with the level energies [see Fig. 1(a)], a more general approach is needed to incorporate the full $\mathbf{k} \cdot \mathbf{p}$ results.

Nevertheless, there is no guarantee that including the full subband dispersions would explain the experimentally observed redshift. Another possibility is that the 60:40 
split between conduction and valence band offset is not perfectly constant with composition. As with the band gap, this might only amount to a minor correction for square wells, but could still have a significant impact on PQW behavior.

Figure 3(b) demonstrates the importance of such issuesnot only do they affect the absorption peak position, but they can affect the linewidth as well. The dashed lines of Fig. 3(b) exhibit a broadening at higher temperature, yet a constant, phenomenological value $\Gamma_{j}=1 \mathrm{meV}=0.24 \mathrm{THz}$ was used in the simulations. This suggests that distortions to the band edge profile could give rise to an inhomogeneous broadening effect. At low temperatures there is a single multisubband plasmon mode-like an ideal PQW-but at higher temperatures this mode splits into several nearby modes, giving an apparent broadening.

It remains unclear how much this contributes to the experimentally observed linewidth. Other key factors could include electron-electron scattering or scattering with ionized impurities, phonons, or the alloy itself [32]. However, the above observation suggests that further reduction of the linewidth - beyond what we already observe in Fig. 2(c) could be possible by further refining the experimentally implemented potential profile. This would require better modeling, incorporating the full $\mathbf{k} \cdot \mathbf{p}$ picture, as well as improved knowledge of key $\mathrm{Al}_{x} \mathrm{Ga}_{1-x}$ As material properties. Other modifications, such as increased barrier width to reduce impurity scattering, could also be beneficial, depending on which factors are currently limiting the linewidth.

In conclusion, we have demonstrated the growth of highquality harmonic oscillator arrays in $\mathrm{Al}_{x} \mathrm{Ga}_{1-x}$ As alloys by faithfully tracking the designed composition profile during MBE growth. The system oscillates at $3 \mathrm{THz}$, from $10 \mathrm{~K}$ up to room temperature, exhibiting narrow linewidths. In line with Ref. [2], the oscillation frequency is largely independent of doping and temperature, despite the presence of significant Hartree potentials. Discrepancies between the model and the results point toward key uncertainties in the understanding of such systems.

The very high quality of these PQW systems opens up several new vistas. Combined with terahertz optical cavities, these systems could already enable the implementation of high-quality terahertz polariton devices operating above liquid-nitrogen temperatures. Even in multipass geometries similar to those employed in this Letter, PQW stacks with low nonradiative scattering rates could potentially be used to explore regimes in which radiative losses play a more prominent role - analogous to demonstrations of (super)radiantly limited linewidths [33,34]. Another very appealing research direction would be the demonstration of ISB transitions in the subterahertz region (200-600 GHz). This would enable the study of the transport of a quantum hall system in strong coupling with a microwave cavity, something that currently is possible only with Landau level systems at extremely low temperatures.

We thank Ludovic Largeau for sample observation by scanning tunneling electron microscopy, and we thank P. Filloux and S. Suffit for cleanroom help at Paris Centre Cleanrooms. This research was undertaken thanks in part to funding from the Canada First Research Excellence Fund, as well as the Natural Sciences and Engineering Research Council of Canada (NSERC). We acknowledge financial support from the European Union FET-Open Grant MIRBOSE (737017) and from the French National Research Agency (project TERASEL ANR 18-CE240013). This work was partly supported by the French RENATECH network.

*raffaele.colombelli@u-psud.fr

tzig.wasilewski@uwaterloo.ca

[1] W. Kohn, Phys. Rev. 123, 1242 (1961).

[2] L. Brey, N. F. Johnson, and B. I. Halperin, Phys. Rev. B 40, 10647 (1989).

[3] C. Sirtori, S. Barbieri, and R. Colombelli, Nat. Photonics 7, 691 (2013).

[4] L. Bosco, M. Franckié, G. Scalari, M. Beck, A. Wacker, and J. Faist, Appl. Phys. Lett. 115, 010601 (2019).

[5] S. Fathololoumi, E. Dupont, C. W. I. Chan, Z. R. Wasilewski, S. R. Laframboise, D. Ban, A. Mátyás, C. Jirauschek, Q. Hu, and H. C. Liu, Opt. Express 20, 3866 (2012).

[6] X. G. Guo, J. C. Cao, R. Zhang, Z. Y. Tan, and H. C. Liu, IEEE J. Sel. Top. Quantum Electron. 19, 8500508 (2013).

[7] R. Colombelli and J.-M. Manceau, Phys. Rev. X 5, 011031 (2015).

[8] S. De Liberato and C. Ciuti, Phys. Rev. Lett. 102, 136403 (2009).

[9] C. Ciuti, G. Bastard, and I. Carusotto, Phys. Rev. B 72, 115303 (2005).

[10] Y. Todorov, A. M. Andrews, R. Colombelli, S. De Liberato, C. Ciuti, P. Klang, G. Strasser, and C. Sirtori, Phys. Rev. Lett. 105, 196402 (2010).

[11] A. Delteil, A. Vasanelli, Y. Todorov, C. Feuillet Palma, M. Renaudat St-Jean, G. Beaudoin, I. Sagnes, and C. Sirtori, Phys. Rev. Lett. 109, 246808 (2012).

[12] M. Geiser, F. Castellano, G. Scalari, M. Beck, L. Nevou, and J. Faist, Phys. Rev. Lett. 108, 106402 (2012).

[13] R. C. Miller, A. C. Gossard, D. A. Kleinman, and O. Munteanu, Phys. Rev. B 29, 3740 (1984).

[14] E. G. Gwinn, R. M. Westervelt, P. F. Hopkins, A. J. Rimberg, M. Sundaram, and A. C. Gossard, Phys. Rev. B 39, 6260 (1989).

[15] P. F. Hopkins, A. J. Rimberg, E. G. Gwinn, R. M. Westervelt, M. Sundaram, and A. C. Gossard, Appl. Phys. Lett. 57, 2823 (1990).

[16] T. Sajoto, J. Jo, M. Santos, and M. Shayegan, Appl. Phys. Lett. 55, 1430 (1989).

[17] A. Wixforth, M. Sundaram, K. Ensslin, J. H. English, and A. C. Gossard, Phys. Rev. B 43, 10000 (1991).

[18] A. V. Trifonov, E. D. Cherotchenko, J. L. Carthy, I. V. Ignatiev, A. Tzimis, S. Tsintzos, Z. Hatzopoulos, 
P. G. Savvidis, and A. V. Kavokin, Phys. Rev. B 93, 125304 (2016).

[19] B. Paulillo, J. M. Manceau, L. H. Li, A. G. Davies, E. H. Linfield, and R. Colombelli, Appl. Phys. Lett. 108, 101101 (2016).

[20] C. Deimert and Z. R. Wasilewski, J. Cryst. Growth 514, 103 (2019).

[21] D. E. Aspnes, W. E. Quinn, M. C. Tamargo, M. A. A. Pudensi, S. A. Schwarz, M. J. S. P. Brasil, R. E. Nahory, and S. Gregory, Appl. Phys. Lett. 60, 1244 (1992).

[22] See Supplemental Material at http://link.aps.org/supplemental/ 10.1103/PhysRevLett.125.097403 for further details of the terahertz transmission spectroscopy, the absorption spectrum calculation in Fig. 3(b), and the photoluminescence excitation experiment.

[23] M. Załużny and C. Nalewajko, Phys. Rev. B 59, 13043 (1999).

[24] E. R. Weber, R. K. Willardson, H. Liu, and F. Capasso, Intersubband Transitions in Quantum Wells: Physics and Device Applications (Academic Press, New York, 1999).

[25] I. Vurgaftman, J. R. Meyer, and L. R. Ram-Mohan, J. Appl. Phys. 89, 5815 (2001).
[26] Z. R. Wasilewski, M. M. Dion, D. J. Lockwood, P. Poole, R. W. Streater, and A. J. SpringThorpe, J. Appl. Phys. 81, 1683 (1997).

[27] G. Pegolotti, A. Vasanelli, Y. Todorov, and C. Sirtori, Phys. Rev. B 90, 035305 (2014).

[28] F. Alpeggiani and L. C. Andreani, Phys. Rev. B 90, 115311 (2014).

[29] S. Birner, T. Zibold, T. Andlauer, T. Kubis, M. Sabathil, A. Trellakis, and P. Vogl, IEEE Trans. Electron Devices 54, 2137 (2007).

[30] G. Bastard, E. E. Mendez, L. L. Chang, and L. Esaki, Phys. Rev. B 26, 1974 (1982).

[31] B. Askenazi, A. Vasanelli, A. Delteil, Y. Todorov, L. C. Andreani, G. Beaudoin, I. Sagnes, and C. Sirtori, New J. Phys. 16, 043029 (2014).

[32] T. Unuma, M. Yoshita, T. Noda, H. Sakaki, and H. Akiyama, J. Appl. Phys. 93, 1586 (2003).

[33] Q. Zhang, T. Arikawa, E. Kato, J. L. Reno, W. Pan, J. D. Watson, M. J. Manfra, M. A. Zudov, M. Tokman, and M. Erukhimova et al., Phys. Rev. Lett. 113, 047601 (2014).

[34] T. Laurent, Y. Todorov, A. Vasanelli, A. Delteil, C. Sirtori, I. Sagnes, and G. Beaudoin, Phys. Rev. Lett. 115, 187402 (2015). 